\title{
RNA-activated DNA cleavage by the Type III-B CRISPR-Cas effector complex
}

\author{
Michael A. Estrella, Fang-Ting Kuo, and Scott Bailey \\ Department of Biochemistry and Molecular Biology, Bloomberg School of Public Health, Johns Hopkins University, \\ Baltimore, Maryland 21205, USA
}

\begin{abstract}
The CRISPR (clustered regularly interspaced short palindromic repeat) system is an RNA-guided immune system that protects prokaryotes from invading genetic elements. This system represents an inheritable and adaptable immune system that is mediated by multisubunit effector complexes. In the Type III-B system, the Cmr effector complex has been found to cleave ssRNA in vitro. However, in vivo, it has been implicated in transcription-dependent DNA targeting. We show here that the Cmr complex from Thermotoga maritima can cleave an ssRNA target that is complementary to the CRISPR RNA. We also show that binding of a complementary ssRNA target activates an ssDNA-specific nuclease activity in the histidine-aspartate (HD) domain of the Cmr2 subunit of the complex. These data suggest a mechanism for transcription-coupled DNA targeting by the Cmr complex and provide a unifying mechanism for all Type III systems.
\end{abstract}

[Keywords: CRISPR-Cas; CMR; RAMP; nuclease; RNA]

Supplemental material is available for this article.

Received October 20, 2015; revised version accepted January 11, 2016.

Clustered regularly interspaced short palindromic repeats (CRISPRs) and their associated (Cas) proteins form the basis of an inheritable and adaptable RNA-guided immune system in prokaryotes (for a recent review, see van der Oost et al. 2014). CRISPR loci are composed of repeat sequences separated by variable spacer sequences that are derived from foreign genetic elements (Bolotin 2005; Mojica et al. 2005; Pourcel 2005; Barrangou et al. 2007). Transcripts from CRISPR loci are processed to generate short CRISPR RNAs (crRNA) (Brouns et al. 2008; Carte et al. 2008; Deltcheva et al. 2011). The crRNA are then incorporated into effector complexes that identify and destroy invading nucleic acid that is complementary to the guide region of the crRNA (Brouns et al. 2008; Hale et al. 2009; Jinek et al. 2012; Gasiunas et al. 2012).

CRISPR-Cas systems are organized into three types (Types I, II, and III) and at least 10 subtypes (Makarova et al. 2011). Each type has a distinct mechanism for processing CRISPR transcripts and for target destruction (Makarova et al. 2011) and is distinguished by a signature protein: Cas3 in Type I, Cas9 in Type II, and Cas 10 in Type III. The effector complexes in Type I systems recognize their DNA targets and then recruit the Cas3 protein to degrade the invading DNA using its histidine-aspartate (HD) nuclease domain (Brouns et al. 2008; Westra et al. 2012; Mulepati and Bailey 2013; Sinkunas et al. 2013). Type II systems also target DNA, but recognition and

Corresponding author: scott.bailey@jhu.edu Article published online ahead of print. Article and publication date are online at http://www.genesdev.org/cgi/doi/10.1101/gad.273722.115. cleavage are mediated by the same effector complex, Cas9 (Gasiunas et al. 2012; Jinek et al. 2012). The Type III systems bind and cleave ssRNA (Hale et al. 2009, 2012; Zhang et al. 2012; Staals et al. 2013, 2014; Tamulaitis et al. 2014; Zebec et al. 2014; Samai et al. 2015) and transcriptionally active DNA (Deng et al. 2013; Goldberg et al. 2014; Samai et al. 2015). The Type III systems can be further classified into Type III-A and Type III-B subtypes. Both subtypes contain a Cas10 family protein: Cmr2 in Type III-B and Csm1 in Type III-A systems (Makarova et al. 2011). Despite mechanistic differences, the Type I and Type III effector complexes share similar structures and likely evolved from a common ancestor (Jackson and Wiedenheft 2015).

The Cmr complexes from the Type III-B systems of Pyrococcus furiosus, Thermus thermophiles, and Sulfolobus solfataricus have all been characterized in vitro (Hale et al. 2009; Zhang et al. 2012; Staals et al. 2013). The Cmr effector complex consists of six proteins (Cmr1-6) and a crRNA. The crRNA is comprised of 8 nucleotides (nt) of repeat sequence at its $5^{\prime}$ end (the $5^{\prime}$ handle) and typically 30-40 nt of invader-derived guide sequence at its $3^{\prime}$ end (Carte et al. 2008; Hale et al. 2009; Staals et al. 2013). Multiple Cmr4 and Cmr5 subunits form a helical filament around the crRNA, which is capped by $\mathrm{Cmr} 2$ and $\mathrm{Cmr} 3$

(C) 2016 Estrella et al. This article is distributed exclusively by Cold Spring Harbor Laboratory Press for the first six months after the full-issue publication date (see http://genesdev.cshlp.org/site/misc/terms.xhtml). After six months, it is available under a Creative Commons License (Attribution-NonCommercial 4.0 International), as described at http:// creativecommons.org/licenses/by-nc/4.0/. 
at its $5^{\prime}$ end and by Cmr6 and Cmrl at its $3^{\prime}$ end (Spilman et al. 2013; Staals et al. 2013; Benda et al. 2014; Osawa et al. 2015; Taylor et al. 2015). Cmr4 serves as a catalytic subunit (Benda et al. 2014; Ramia et al. 2014a; Zhu and Ye 2015), cleaving paired ssRNA targets at regular 6-nt intervals (Staals et al. 2013; Hale et al. 2014). Cmr2 contains a predicted HD nuclease domain (Makarova et al. 2011), although this domain is dispensable for RNA target cleavage by the Cmr complex (Cocozaki et al. 2012; Staals et al. 2013). The Csm complexes from the Type III-A systems have also been characterized in vitro. They have a structure similar to that of the Cmr complex (Rouillon et al. 2013; Staals et al. 2014) and likewise cleave complementary RNA at multiple sites (Staals et al. 2014; Tamulaitis et al. 2014; Samai et al. 2015) but have also been shown to cleave DNA in a transcription-coupled manner (Samai et al. 2015).

In cells, genetic experiments have implicated the Cmr complex in targeting transcriptionally active DNA (Deng et al. 2013), but the mechanism of this activity and the identity of the DNA nuclease active site are unknown. We therefore undertook a biochemical analysis of the Cmr complex to determine whether and how it can cleave DNA.

\section{Results}

Reconstitution of the Cmr complex from Thermotoga maritima

The genome of T. maritima MSB8 contains three Cas modules (one each of Types I-B, III-A, and III-B) and eight CRISPR loci (Supplemental Fig. S1). Each CRISPR loci contains the same or a very similar repeat sequence, suggesting that the three Cas modules can likely use crRNA processed from any of the CRISPR loci. The Type III-B module encodes six proteins (Cmr1-6). To determine whether these six proteins can assemble into a Cmr complex, we cloned, expressed, and purified each T. maritima Cmr protein in an Escherichia coli expression system (Fig. 1A). The recombinant proteins were mixed, and the mixture was purified over a nickel column (Cmr6 was N-terminally hexahistidine-tagged; all of the other proteins were untagged) in either the presence or absence of a syn-

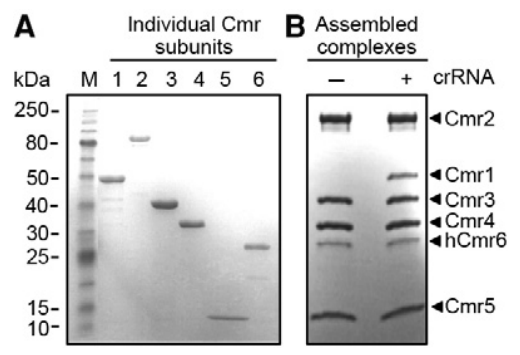

Figure 1. Assembly of the T. maritima Cmr complex. SDS-polyacrylamide gels, stained with Coomassie blue, of individually purified Cmr subunits $(A)$ and affinity-purified Cmr complex $(B)$ with and without crRNA8.3. thetic crRNA8.3 (which corresponds to the third crRNA encoded by CRISPR locus 8). In the absence of crRNA, all but one of the Cmr proteins, Cmrl, purified as a complex, whereas, in the presence of crRNA, all six Cmr proteins purified as a complex (Fig. 1B). Weak association between Cmrl and the Cmr complex has been noted in both T. thermophilus (Staals et al. 2013) and P. furiosus (Benda et al. 2014).

We next asked whether the organization of the T. maritima Cmr complex is similar to $\mathrm{Cmr}$ complexes from other organisms. To test this, we performed a series of pull-down experiments with combinations of T. maritima $\mathrm{Cmr}$ proteins. The results of these experiments are presented in Supplemental Figure S2. The interactions between subunits observed in the T. maritima Cmr complex agrees with the interactions observed between subunits of other Cmr complexes by electron microscopy, cross-linking, and X-ray crystallography (Spilman et al. 2013; Staals et al. 2013; Benda et al. 2014; Osawa et al. 2015; Taylor et al. 2015), suggesting that the T. maritima Cmr complex has the same subunit architecture as other Cmr complexes.

\section{The T. maritima Cmr complex cleaves ssRNA}

In vitro, the Cmr complexes from $P$. furiosus, $S$. solfataricus, and T. thermophilus cleave ssRNA that is complementary to the crRNA (Hale et al. 2009; Zhang et al. 2012; Staals et al. 2013). To determine the substrate specificity of the $T$. maritima Cmr complex, we incubated the complex with crRNA8.3 and a selection of nucleic acid targets that were radiolabeled at their $5^{\prime}$ ends $\left(5^{\prime}\right.$ labeled). Reactions were incubated for $10 \mathrm{~min}$ at $80^{\circ} \mathrm{C}$, the optimal growth temperature of T. maritima (Huber et al. 1986), and the products were analyzed by denaturing PAGE followed by autoradiography. Noncomplementary ssRNA, complementary ssDNA, and dsRNA were not cleaved (Fig. 2A). Only complementary ssRNA (the ssRNA8.3 target) was cleaved, with the cleavage site located 14 nt from the $3^{\prime}$ end of the paired crRNA (Fig. 2). Cleavage appeared to be sequence-independent, as a ssRNA target complementary to a crRNA with a different sequence (crRNA8.4 and ssRNA8.4) also resulted in the same 14-nt cleavage product (Fig. 2A). Cleavage also required that the $5^{\prime}$ handle was intact and had the correct repeat-derived sequence (Fig. 2B), in agreement with results from the P. furiosus Cmr complex (Hale et al. 2012), and, like other Type III complexes, was dependent on the presence of magnesium or manganese ions (Supplemental Fig. S3A; Hale et al. 2009; Zhang et al. 2012; Staals et al. 2013, 2014; Tamulaitis et al. 2014; Samai et al. 2015).

Other Cmr complexes can cleave their RNA targets at up to five sites that are separated by 6-nt intervals (Staals et al. 2013; Hale et al. 2014). To determine the frequency and spacing of target cleavage by the T. maritima $\mathrm{Cmr}$ complex, we monitored cleavage of a $5^{\prime}$ labeled ssRNA8.3 target as a function of time. The results show that the target was cleaved at four sites (sites 1-4), each separated by 6 nt, producing 32-, 26-, 20-, and 14-nt products (Fig. 2C). Cleavage at site 1 is minimal compared with the other 


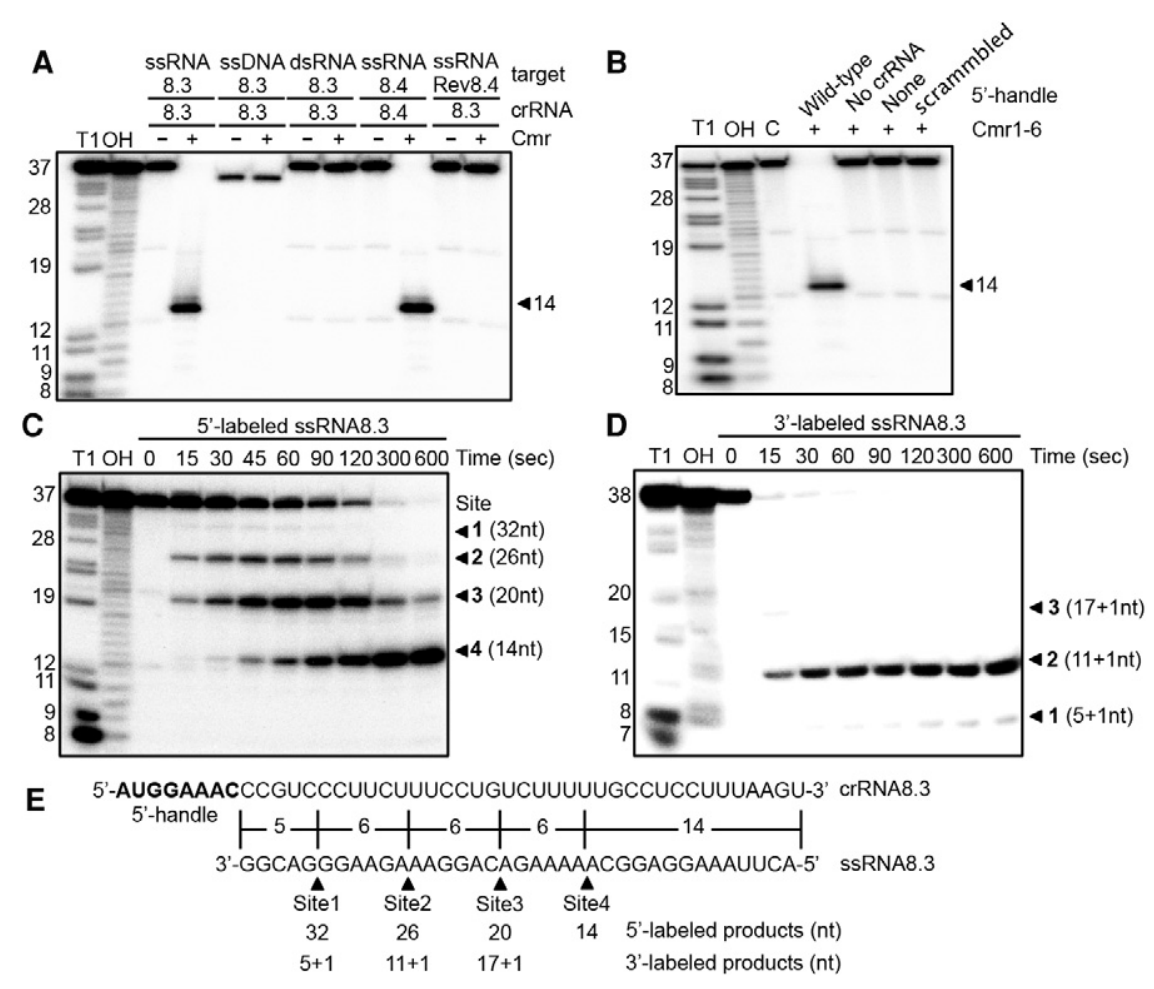

Figure 2. The T. maritima Cmr complex cleaves ssRNA. $(A)$ The indicated 5 labeled substrates were incubated with $(+)$ or without $(-)$ the Cmr complex, and the products were resolved on a denaturing polyacrylamide gel. $(B)$ Requirement of the crRNA and determinant of its repeat-derived $5^{\prime}$ handle. $(C, D) 5^{\prime}$ and $3^{\prime}$ labeled ssRNA8.3 targets were incubated with the Cmr complex for the indicated times and resolved on denaturing polyacrylamide gels. In all panels, "T1" denotes the $\mathrm{T} 1$ ladder, and "OH" denotes the hydroxide ladder. $(E)$ Schematic depicting the cleavage sites on the ssRNA8.3 target in relation to crRNA8.3. The sizes of the cleavage products are indicated. Note that the $3^{\prime}$ end labeling reaction added one additional nucleotide to the $3^{\prime}$ end of the RNA target. three sites, which was also observed with the $P$. furiosus and T. thermophilus Cmr complexes (Staals et al. 2013; Hale et al. 2014). The appearance and disappearance of the cleavage products over time suggests that the target is cleaved sequentially, starting predominantly at site 2 and then proceeding to site 3 and then to site 4 (Fig. 2E). After $\sim 5 \mathrm{~min}$, the target is almost completely cleaved to a single prominent 14-nt product, which is much faster than observed with $P$. furiosus and T. thermophilus $\mathrm{Cmr}$ complexes (Hale et al. 2009; Staals et al. 2013). To confirm that cleavage is sequential, we repeated this experiment but used a 3' labeled target. In this experiment, we observed a single prominent 12-nt product (Fig. 2D), corresponding to cleavage at site 2 (note that the $3^{\prime}$ end labeling reaction added one additional nucleotide to the 3 ' end of the RNA target), suggesting that cleavage primarily begins at site 2 .

To gain further insights into the determinants for RNA cleavage, we tested activity on RNA targets that would result in base-pairing-disrupting mismatches when paired with crRNA8.3. RNA targets that result in mismatches at position 20 and/or position 21, which flank the scissile phosphate at site 3, do not inhibit cleavage of the target at any site. In fact, cleavage of these mismatched targets appears to be enhanced relative to the fully complementary target, as, with these targets, we observed an increased proportion of the site 4 product relative to the fully complementary target (Supplemental Fig. S4A, experiments 1-4). Thus, perfect complementarity is not required at site 3, suggesting that RNA cleavage by the Cmr complex, like the related Csm complexes (Staals et al. 2014; Tamulaitis et al. 2014), is not strictly dependent on complete complementarity between the crRNA and RNA target.
It has been shown that the 2 '-hydroxyl group adjacent to the scissile phosphate is crucial for the RNA cleavage activity of a chimeric Cmr complex reconstituted from Archaeoglobus fulgidus Cmr1-3 and P. furiosus Cmr4-6 (Osawa et al. 2015). We confirmed the importance of this hydroxyl group to cleavage by the T. maritima $\mathrm{Cmr}$ complex. An ssRNA8.3 target containing a 2'-deoxyribose at position 20 was not cleaved at site 3 (Supplemental Fig. S4A, experiment 5), but, in a control experiment, a target with a $2^{\prime}$-deoxyribose at position 21 was cleaved at site 3 (Supplemental Fig. S4A, experiment 6). Furthermore, when any of the four cleavage sites were blocked with a 2 -deoxyribose, we observed no cleavage at that site (Supplemental Fig. S4B, experiments 7-11). A modified ssRNA8.3 target (ssRNA8.3*) that contained 2'-deoxyribose base substitutions at each of the four cut sites (positions 14, 20, 26, and 32) was not cleaved by the Cmr complex at any site (Supplemental Fig. S4B, experiment 12). To confirm that the $2^{\prime}$-deoxyribose modifications in ssRNA8.3* do not interfere with binding to the Cmr complex, we performed competition experiments. Here, we monitored cleavage of the $5^{\prime}$ labeled ssRNA8.3 target using either unlabeled ssRNA8.3* as a specific competitor or ssRNA8.4 as an unspecific competitor. Only the unlabeled crRNA8.3* inhibited cleavage of the target (Supplemental Fig S4C, lane 3), thereby providing support for the Cmr complex binding to crRNA8.3*.

\section{DNA cleavage by the Cmr complex}

Genetic experiments implicate the Cmr complex in targeting transcriptionally active plasmid DNA in cells (Deng et al. 2013). However, Cmr complexes have only 
been observed to cleave RNA and not DNA in vitro (Fig. 2A; Hale et al. 2009; Zhang et al. 2012; Staals et al. 2013) and in vivo (Hale et al. 2012; Zebec et al. 2014). We reasoned that these differing observations could be reconciled if the DNA nuclease activity of the Cmr complex required the presence of a ssRNA transcript. To test this, we incubated the T. maritima Cmr complex with crRNA8.3, an ssRNA8.3 target (mimicking a transcript), and one of two different ssDNA substrates (substrates A and B), which were $5^{\prime}$ labeled (Fig. 3A). We initially screened ssDNA as a potential substrate because Csm1 (which, like Cmr2, is a Cas10 family protein) has ssDNA nuclease activity (Jung et al. 2015) and is the only subunit of a Type III effector complex with known DNA nuclease activity. The Cmr complex cut the DNA substrate weakly at multiple sites only when it and the ssRNA8.3 target were added to the reaction at the same time (Fig. 3B, lanes 4,11 ). No cleavage was observed if the Cmr complex was preincubated with the ssRNA8.3 target for $10 \mathrm{~min}$ before addition of the ssDNA (Fig. 3B, lanes 3,10). This suggested that cleavage of the ssRNA target may inhibit cleavage of the ssDNA substrate. No DNA cleavage was observed in the absence of the Cmr complex, in the absence of the complementary ssRNA8.3 target (Fig. 3B), or with an ssRNA target noncomplementary to crRNA8.3 (Fig. 3B, lanes 7,14). Cleavage was dependent on the presence of manganese ions but not magnesium ions (Supplemental Fig. S3B) and was specific to ssDNA, as no cleavage of either ssRNA or dsDNA was observed under the same reactions conditions (Fig. 3B, lanes 15-18).

To further investigate the effects of RNA cleavage on the DNA cleavage activity of the Cmr complex, we monitored DNA cleavage under two regimes where the ssRNA target is not cut. First, we monitored DNA cleavage using the noncleavable ssRNA8.3* as the RNA target. Second, we monitored DNA cleavage using a Cmr complex that was reconstituted with Cmr4 harboring a D26A mutation (Benda et al. 2014; Ramia et al. 2014a; Zhu and Ye 2015). This variant of the T. maritima Cmr complex specifically binds to (Supplemental Fig. S5, lanes 3,8) but is unable to cleave (Supplemental Fig. S6A, lane 3) a complementary RNA target. Under both of these regimes, cleavage of the ssDNA was greatly enhanced (Fig. 3B, lanes 5,6, $12,13)$, suggesting that cleavage of the RNA target inhibits cleavage of ssDNA by the Cmr complex.

\section{RNA target binding licenses ssDNA cleavage}

Our results suggest that RNA target binding licenses ssDNA cleavage by the Cmr complex. To gain additional insight into this licensing, we investigated RNA cleavage more closely. First, we wished to determine whether the RNA products dissociate from the Cmr complex following cleavage or whether they remain bound. Thus, the Cmr complex pre-equilibrated with an equimolar amount of crRNA8.3 was incubated with either equimolar or twofold or fourfold excess of the ssRNA8.3 target. Cleavage was monitored over time by denaturing PAGE followed by autoradiography. If RNA products did not dissociate from the complex following cleavage, then, in reactions with target in excess, the amount of target cleaved should equal the amount of Cmr complex in the reaction. However, at all ratios of Cmr complex to target tested, $100 \%$ of the ssRNA8.3 target was cleaved (Fig. 4A), indicating that the RNA products dissociate from the complex following cleavage. In a second set of experiments, the Cmr complex, pre-equilibrated with 50 nM crRNA8.3 (1:1 stoichiometry), was incubated with $100 \mathrm{nM}$ target ssRNA8.3 and $1 \mathrm{nM}$ ssDNA substrate A (5' radiolabeled). Cleavage of the DNA was monitored over time until the accumulation of product had plateaued (Fig. 4B). Note that, under these conditions (100 nM ssRNA8.3 target), the DNA cleavage activity of the $\mathrm{Cmr}$ complex was more robust than in previous experiments, where the concentration of the ssRNA8.3 target was much lower (1 nM). Once the accumulation of product had plateaued, fresh complementary (ssRNA8.3) or, in a control experiment, noncomplementary (ssRNA8.4) RNA targets were added to the

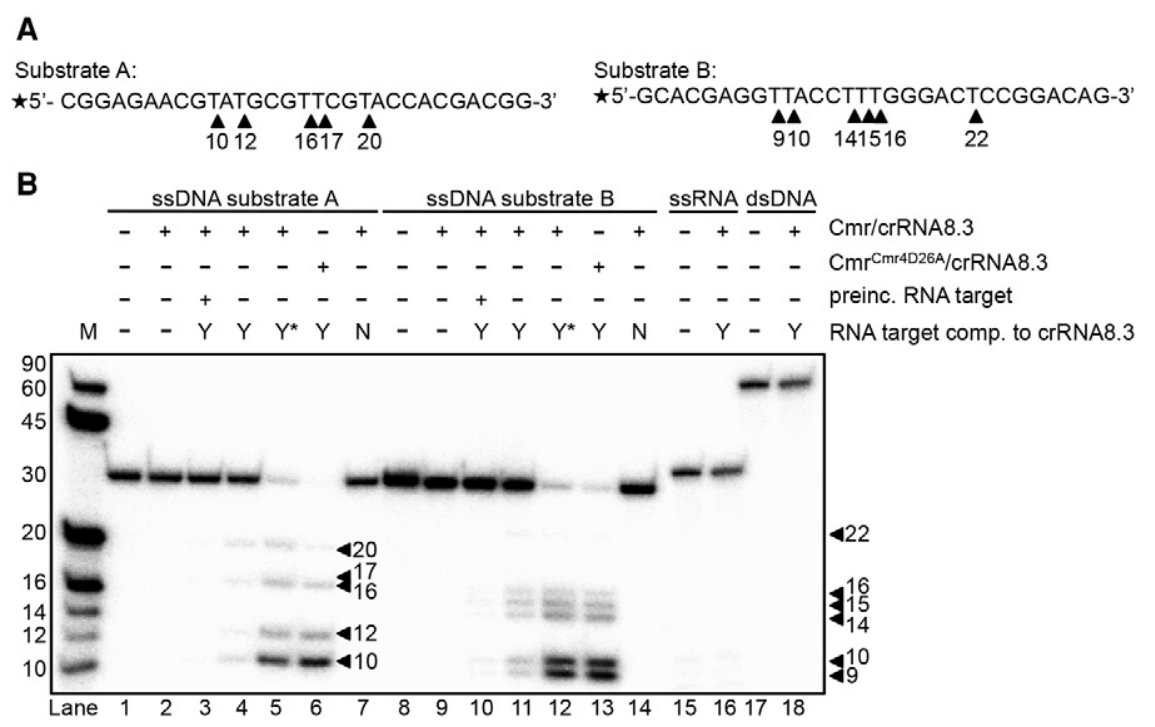

Figure 3. DNA cleavage by the Cmr complex. (A) Schematic of DNA substrates A and $\mathrm{B}$. Arrowheads indicate the mapping of the cleavage sites onto the sequences. (B) 5' labeled ssDNA substrates (substrates $A$ and $B$ ), an ssRNA (whose sequence is equivalent to ssDNA substrate A), or a dsDNA (substrate A annealed to a complementary oligol were incubated with the Cmr complex (or a variant Cmr complex containing Cmr4 D26A) in the presence of ssRNA8.3 targets and then analyzed by denaturing PAGE. An asterisk indicates the use of the noncleavable ssRNA8.3* target, and " $\mathrm{M}$ " denotes the marker lane. 
A

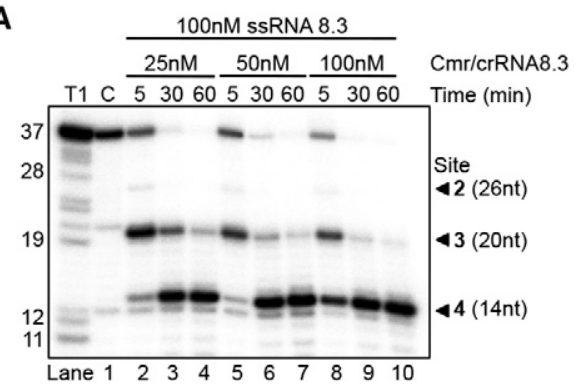

B

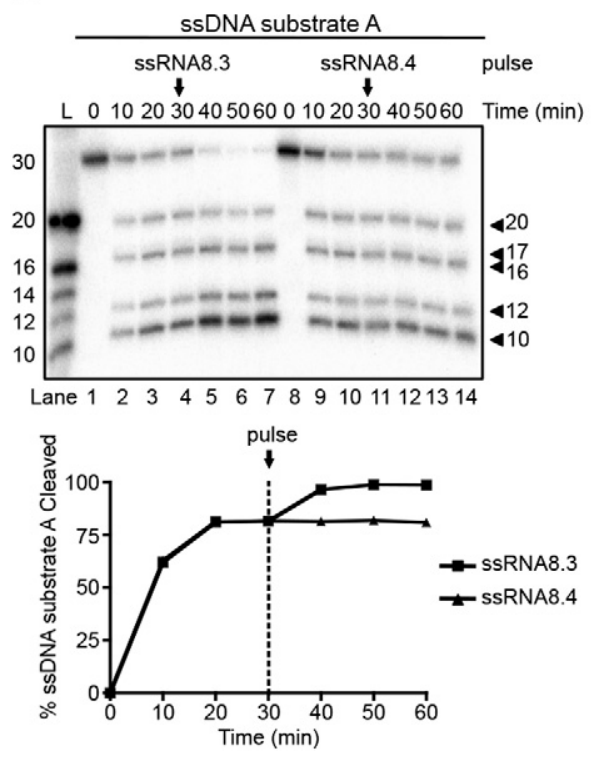

Figure 4. RNA turnover by the Cmr complex. (A) $5^{\prime}$ labeled ssRNA8.3 target was incubated with the Cmr complex in the presence of unlabeled crRNA8.3 at the indicated concentrations, and cleavage was monitored over time. "T1" denotes the T1 ladder, and "C" denotes a control lane containing only the labeled ssRNA8.3 target. $(B) 5^{\prime}$ labeled ssDNA substrate A was incubated with the unlabeled ssRNA8.3, the Cmr complex, and crRNA8.3 for $30 \mathrm{~min}$ before either fresh ssRNA8.3 or ssRNA8.4 was added to the reaction (pulse), which was then monitored for a further $30 \mathrm{~min}$. Samples were analyzed by denaturing PAGE. Below the gel panel is a quantification of the cleavage activity over time. The point at which the pulse was added is indicated.

reactions, and we continued to monitor DNA cleavage (Fig. 4B). Addition of fresh complementary ssRNA8.3 reinitiated cleavage of the ssDNA, whereas the noncomplementary ssRNA8.4 had no effect, suggesting that the RNA products had dissociated from the complex. If the RNA products are not released after cleavage, then addition of fresh target should have no effect. Together, these data indicate that the presence of an intact ssRNA target complementary to the crRNA activates a ssDNA nuclease activity in the Cmr complex. Cleavage of the ssRNA target and dissociation of the resulting fragments then prevent DNA cleavage.

In Type III-A systems, base pairing between the $5^{\prime}$ handle of the crRNA and the $3^{\prime}$ flanking sequence of the RNA target has been shown to have no effect on RNA target cleavage, but it inhibits cleavage of the DNA (Marraffini and Sontheimer 2010; Samai et al. 2015). We tested the effects of complementarity to the $5^{\prime}$ handle on RNA and DNA cleavage by the T. maritima $\mathrm{Cmr}$ complex and found that the RNA was cleaved, albeit to a lesser extent (Supplemental Fig. S6A, lane 5), and that cleavage of DNA was almost undetectable (Supplemental Fig. S6B, lane 3). Thus, activation of DNA cleavage by the Cmr complex also requires a lack of complementarity between the RNA target and the crRNA $5^{\prime}$ handle.

\section{Sequence specificity for the ssDNA target}

The Cmr complex was able to cleave two ssDNA substrates with distinct sequences, suggesting that the ssDNA nuclease activity of the Cmr complex is not sequence-specific. However, cleavage of each substrate produced a distinct band pattern (Fig. 3B), indicating some sequence preference at the sites of cleavage. To investigate this further, we mapped the length of the cleavage products for the two different ssDNA substrates (Fig. 3A). This mapping revealed that the $\mathrm{Cmr}$ complex cleaved the DNA after every thymidine and not after any other nucleotide. Thymidine specificity was further verified using a series of DNA substrates (derived from substrate A) where cleavage was blocked at the sites where thymidines were replaced with adenosine (Fig. 5A). A ssDNA substrate completely lacking thymidine was not cleaved by the Cmr complex (Fig. 5A).

In addition to the observed thymidine specificity, cleavage between a thymidine and a cytidine appeared to be less efficient than cleavage between a thymidine and any other nucleotide (see the 17-nt product of substrate A and the 22-nt produce of substrate B in Fig. 3B). Substrate A contains a single thymidine-cytidine dinucleotide sequence, located at positions 17-18 (Fig. 3A). We mutated this cytidine $(\mathrm{C} 18)$ to each of the other $3 \mathrm{nt}$ and monitored cleavage of the modified DNA substrate. Cleavage at position 17 of these modified substrates was more robust than in substrate A (Fig. 5B), thus confirming that cleavage between a thymidine and a cytidine is less efficient than cleavage between thymidine and any other nucleotide.

\section{Cleavage of ssDNA in the context of dsDNA}

To determine whether the T. maritima Cmr complex can cleave ssDNA in the context of dsDNA, we generated a series of dsDNA substrates that contained mismatched "bubble" regions. The "bubble" regions ranged in size from 2 to $10 \mathrm{nt}$ and consisted of a polythymidine sequence on one strand (the $\mathrm{T}$ strand) and a polycytidine sequence on the opposite strand (the C strand) (Fig. 5C). The double-stranded regions of these substrates were designed such that they did not melt at the reaction temperature, $80^{\circ} \mathrm{C}$. The "bubble" substrates (labeled at the $5^{\prime}$ end of their $\mathrm{T}$ strands) were incubated with the T. maritima Cmr complex crRNA8.3 and an ssRNA8.3 target and then analyzed by denaturing PAGE and autoradiography. Each of the substrates was cleaved multiple times within 


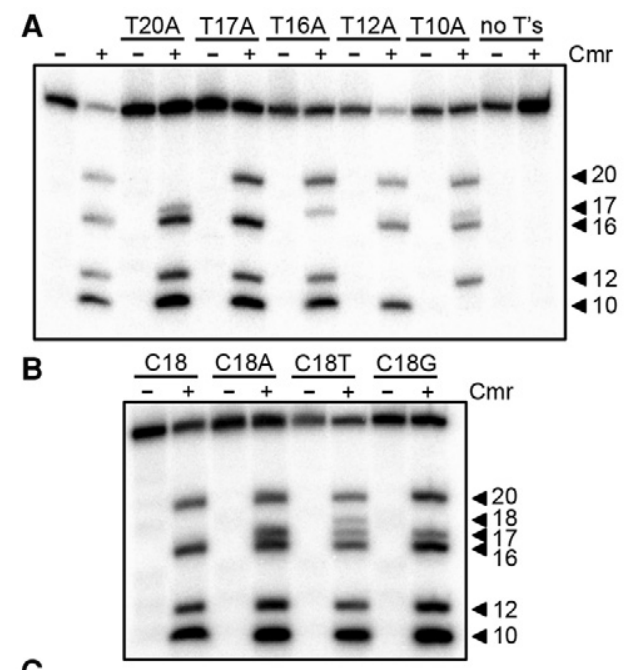

C

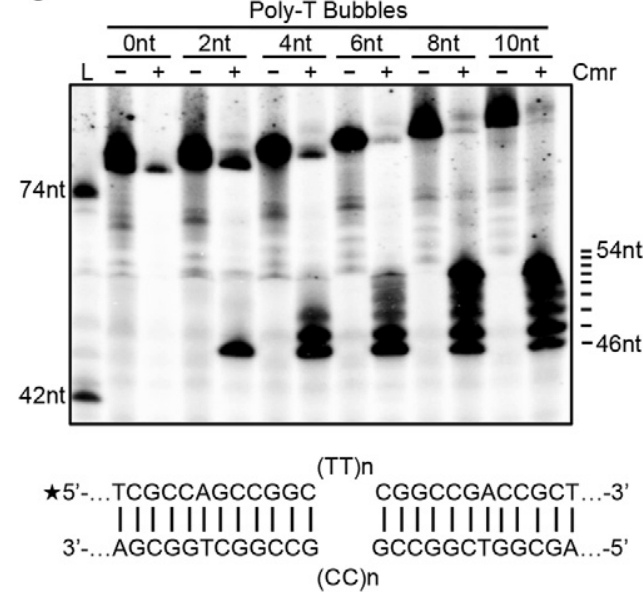

Figure 5. Determinants for DNA cleavage by the T. maritima Cmr complex. (A) $5^{\prime}$ labeled ssDNA substrates with the indicated thymidine-to-adenosine substitutions were incubated with $(+)$ or without (-) the Cmr complex and analyzed by denaturing PAGE. (B) $5^{\prime}$ labeled ssDNA substrates with the indicated substitutions at position 18 were incubated with $(+)$ or without $(-)$ the $\mathrm{Cmr}$ complex and analyzed by denaturing PAGE. $(C)$ dsDNA substrates containing 2-, 4-, 6-, 8-, and 10-nt "bubbles" were incubated with the Cmr complex and analyzed by denaturing PAGE. Below the gel is a schematic of the "bubble" substrate.

the bubble region (Fig. 5C), suggesting that the Cmr complex can cleave ssDNA regions as short as $2 \mathrm{nt}$ in the context of dsDNA.

\section{Mutations in the HD domain of Cmr2 abolish ssDNA cleavage}

The ssDNA nuclease activity of isolated Csm1 has been attributed to its HD domain (Jung et al. 2015). We reasoned that the HD domain of Cmr2 might be responsible for the observed ssDNA cleavage activity of the Cmr complex. To test this prediction, we made a mutant $\mathrm{Cmr} 2$ in which two highly conserved residues in the HD motif (His32 and Asp33) were substituted with alanine. The mu- tant Cmr2 (the HD-AA mutant) was then assembled into the Cmr complex and incubated with crRNA8.3 and either a $5^{\prime}$ labeled ssRNA8.3 target or a $5^{\prime}$ labeled ssDNA and an unlabeled ssRNA8.3 target. After a 10-min incubation at $80^{\circ} \mathrm{C}$, the $\mathrm{HD}$-AA mutant complex did not cleave the ssDNA (Fig. 6, lane 3) but did specifically bind (Supplemental Fig. S5, lanes 4,9) and cleave (Fig. 6, lane 7) the ssRNA8.3 target. These observations suggest that the conserved HD motif found in Cmr2 is critical for the ssDNA endonuclease activity of the Cmr complex.

In the Type III-A system of Staphylococcus epidermidis, the GGDD motif in the Palm domain of Csm 1 is required for DNA cleavage and not the HD domain (Hatoum-Aslan et al. 2014; Ramia et al. 2014b; Samai et al. 2015). To test whether the GGDD motif has a role in DNA cleavage by the T. maritima Cmr complex, we made a mutant Cmr2 in which the two highly conserved aspartate residues (Asp585 and Asp586) in the GGDD motif were substituted with alanine. The mutant Cmr2 (the DD-AA mutant) was then assembled into the Cmr complex, and its cleavage activities were assessed as before with the HD-AA mutant. The DD-AA mutant complex failed to cleave ssDNA (Fig. 6, lane 4) but also failed to cleave the complementary ssRNA8.3 target (Fig. 6, lane 8). To further investigate why this mutant failed to cleave the RNA target, we used an electrophoretic mobility shift assay (EMSA) to access its specific binding to a complementary RNA target (Supplemental Fig. S5, lanes 5,10). We found that the DD-AA mutant complex did not bind a complementary RNA target. This suggests that mutation of the GGDD motif in the T. maritima Cmr2 subunit results in a dysfunctional Cmr complex that cannot bind complementary RNA targets and thus cannot be activated for DNA cleavage.

\section{Discussion}

The Cmr complex from the Type III-B CRISPR-Cas system is a crRNA programmed effector complex that

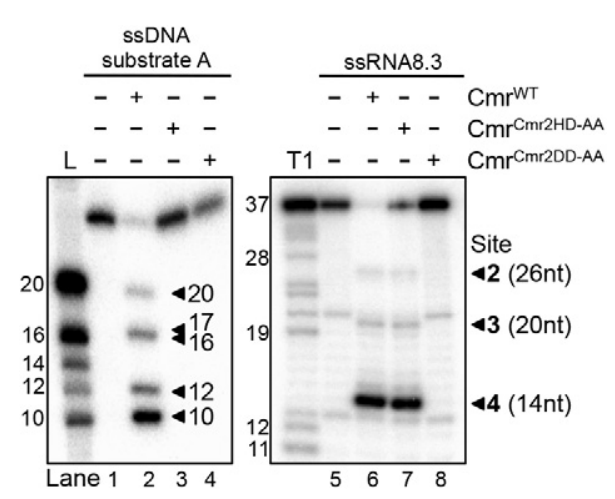

Figure 6. Effects of mutations in Cmr2. $5^{\prime}$ labeled ssRNA8.3 target (right) or $5^{\prime}$ labeled ssDNA substrate A with unlabeled ssRNA8.3 target (left) was incubated with either wild-type (WT) or mutant $\mathrm{Cmr}$ complexes (assembled with Cmr2 HD-AA or Cmr2 DD-AA) and then analyzed by denaturing PAGE. "L" denotes a DNA ladder, and "T1" denotes a T1 RNA ladder. 
cleaves complementary RNA in vitro (Hale et al. 2009; Zhang et al. 2012; Staals et al. 2013) and in vivo (Hale et al. 2012; Zebec et al. 2014). The Cmr complex also protects against plasmid transformation in vivo in a transcription-dependent manner (Deng et al. 2013). All known CRISPR-Cas systems that protect against DNA invasion do so by degrading the invading DNA /Garneau et al. 2010; Westra et al. 2012; Samai et al. 2015), suggesting that DNA targeting by the Cmr complex may also be mediated by DNA cleavage. Here we show that the Cmr complex cleaves ssDNA in the presence of a complementary RNA target (Fig. 3) and discuss the implications of this finding.

We demonstrate that $\mathrm{Cmr}$ proteins from T. maritima assemble into a Cmr complex (Supplemental Fig. S2) with the same subunit interactions as those observed for Cmr complexes from other species (Spilman et al. 2013; Staals et al. 2013; Benda et al. 2014; Osawa et al. 2015; Taylor et al. 2015). The T. maritima Cmr complex specifically cleaves RNA targets that are complementary to crRNA. Cleavage occurs sequentially over four sites separated by 6-nt intervals (Fig. 2C-E) and is catalyzed by the Cmr4 subunit (Supplemental Fig. S6A, lane 3; Benda et al. 2014; Ramia et al. 2014a; Zhu and Ye 2015). As observed previously with a chimeric Cmr complex (Osawa et al. 2015), we demonstrated that cleavage is dependent on the 2'-hydroxyl adjacent to the scissile phosphate (Supplemental Fig. S4). We also found that RNA cleavage by the Cmr complex can tolerate mismatches in the region of a cut site (Supplemental Fig. S4A). A tolerance for mismatches was also observed with Csm complexes (Staals et al. 2014; Tamulaitis et al. 2014), suggesting that both Type III-A and Type III-B systems have flexibility in target specificity.

The Type III CRISPR-Cas systems protect cells from invasion by DNA viruses and plasmids in a transcriptioncoupled manner (Deng et al. 2013; Goldberg et al. 2014; Samai et al. 2015). Here we show that a purified Cmr complex can cleave ssDNA after thymidine nucleotides but only in the presence of a complementary ssRNA target (Fig. 3). The S. solfataricus Cmr complex has been reported to cleave RNA targets at uridine-adenosine dinucleotides (Zhang et al. 2012), suggesting that sequencespecific cleavage of their substrates may be a more general feature of Cmr complexes. RNA-activated DNA cleavage by the Cmr complex provides a basis for the previously observed transcription-coupled DNA targeting. Binding but not cleavage of the ssRNA target was required to activate DNA cleavage (Figs. 3, 4; Supplemental Figs. S5, S6), consistent with immunity against plasmid transformation in the Type III-A system requiring DNA but not RNA cleavage (Samai et al. 2015). Although the structural basis of DNA nuclease activation is unknown, it may involve the conformational rearrangements observed on complementary target binding by cryo-electron microscopy (Taylor et al. 2015). We mapped the active site for ssDNA cleavage to the HD domain of Cmr2 (Fig. 6). Transcription-coupled DNA cleavage may therefore target regions of ssDNA generated by transcription, likely at the displaced nontemplate strand (Fig. 7; Jackson and Wieden-
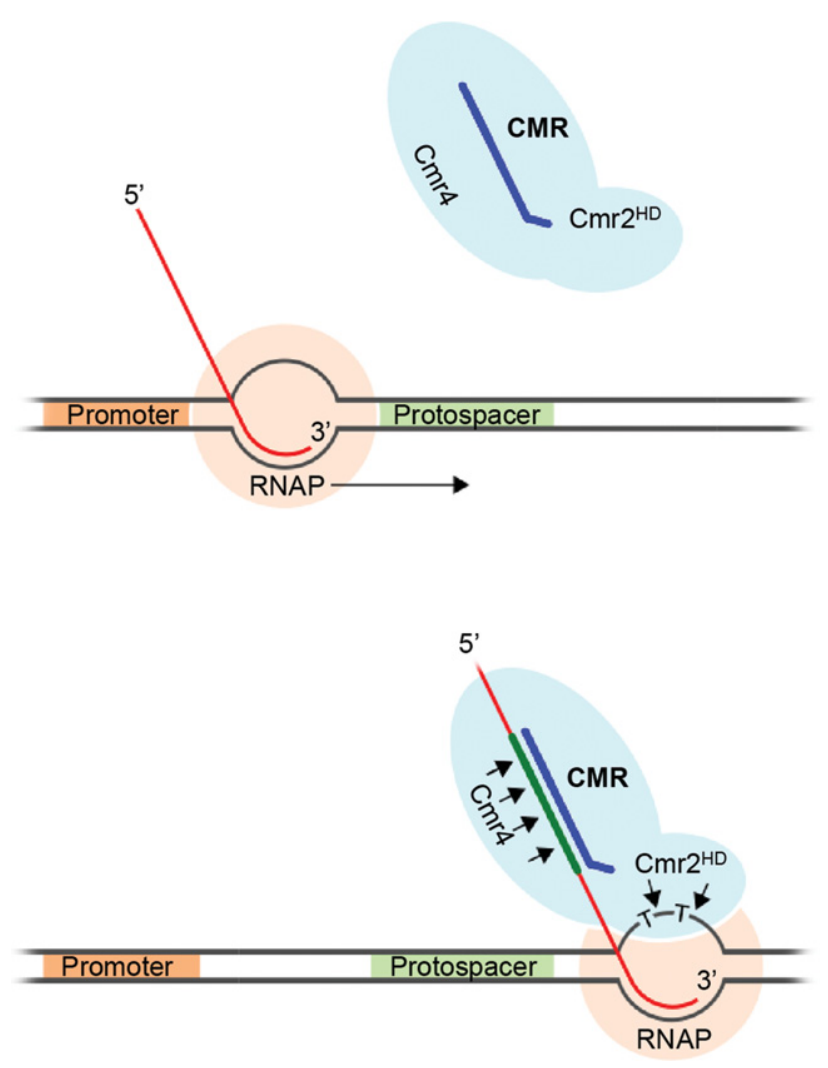

Figure 7. A model for the mode of action of the Type III-B CRISPR-Cas systems. Once RNA polymerase transcribes through the protospacer, the $\mathrm{Cmr}$ complex binds to the nascent transcript through base pairing with the crRNA. Transcript binding activates the DNA and RNA nuclease activities of the Cmr complex. The DNA nuclease activity of the HD domain from $\mathrm{Cmr} 2$ cleaves the displaced nontemplate strand of the transcription bubble at sites $3^{\prime}$ of thymidine nucleotides. The RNA nuclease activity cleaves the mRNA target stepwise through four sites. Activation of the DNA nuclease activity is dependent on the presence of a bound complementary target. Therefore, once the RNA target is cleaved, the DNA nuclease activity is deactivated.

heft 2015; Samai et al. 2015). Accordingly, we observed cleavage of dsDNA that contains a mismatched "bubble" region as small as $2 \mathrm{nt}$ (Fig. $5 \mathrm{C}$ ).

Several subunits of the Type I and Type III CRISPR-Cas complexes share distant phylogenetic relationships (Makarova et al. 2011, 2015), and accumulating structural data suggest that these complexes may have evolved from a common ancestor (for review, see Jackson and Wiedenheft 2015). Our studies indicate that the HD domain of Cmr2 is the domain responsible for DNA cleavage in Type III-B systems. In Type I systems, DNA is also cleaved by an HD domain in the helicase-nuclease Cas3, providing a further evolutionary link between the two systems. Indeed, the HD domains of Cmr2 and Cas3 both depend on transition metals and not magnesium for catalysis, and both cleave ssDNA (Mulepati and Bailey 2011, 2013; Westra et al. 2012; Sinkunas et al. 2013). S. thermophilus Cas3 has also been shown to preferentially cleave after thymidine nucleotides (Sinkunas et al. 2013). 
The immunity against DNA invasion provided by the Type III CRISPR-Cas systems is dependent on COG1517 proteins (Makarova et al. 2012): Csm6 in Type III-A systems (Hatoum-Aslan et al. 2014) and Csx1 in Type III-B systems (Deng et al. 2013). However, none of these proteins stably associates with their respective effector complexes (Hale et al. 2009; Hatoum-Aslan et al. $2014)$ or is required for RNA cleavage by either complex (Fig. 2; Hale et al. 2009; Zhang et al. 2012; Staals et al. 2013, 2014; Tamulaitis et al. 2014; Samai et al. 2015). We also show that cleavage of ssDNA by the RNA-activated Cmr complex does not require the presence of Csx1 (Fig. 3). Likewise, transcription-coupled cleavage of DNA by the Csm complex does not require Csm6 (Samai et al. 2015). Thus, Csx1 and Csm6 do not appear to have a direct role in either RNA or DNA cleavage by the Type III effector complexes. Further biochemical studies are needed to understand the function of these proteins, although, interestingly, sequence analysis suggests that this family of proteins may be nucleases (Makarova et al. 2012).

Two models have been proposed for how a Type III effector complex could identify its DNA target using a crRNA. In the first model, crRNA pairs with the nontemplate strand that has been exposed by a transcription bubble (Deng et al. 2013; Goldberg et al. 2014; Samai et al. 2015). In the second, crRNA pairs with the nascent transcript (Goldberg et al. 2014; Jackson and Wiedenheft 2015). We observed that pairing of a crRNA with an RNA target activates ssDNA cleavage by the Cmr complex, and the sequence specificity of this nuclease activity has no dependence on the crRNA sequence. Also, the $P$. furiosus Cmr complex does not bind ssDNA that is complementary to the crRNA but does bind complementary ssRNA (Hale et al. 2014). Similarly, the S. thermophilus Csm complex binds complementary ssRNA 100 times tighter than ssDNA (Tamulaitis et al. 2014). Together, these data support the second model, in which the Type III effector complex (Cmr or Csm) binds to the nascent transcript (Fig. 7). Once recruited by transcript binding, the ssDNA cleavage activity of the effector complex is activated. A likely substrate for this activity is the displaced ssDNA of the displaced nontemplate strand (Samai et al. 2015) of the downstream transcription bubble (Fig. 7), which has been shown by previous footprinting assays to be more accessible than the template strand in a transcription bubble (Wang and Landick 1997).

The active site for DNA cleavage may vary both between and within Type III-A and Type III-B systems. Through mutagenesis, we mapped the DNA nuclease activity of the Type III-B Cmr complex to the HD motif of Cmr2 (Fig. 6), as initially predicted from sequence analysis (Makarova et al. 2011). The HD domain of the Csm1 protein from the Type III-A system of Thermococcus onnurineus was also found to have DNA nuclease activity (Jung et al. 2015). However, in the Type III-A system of S. epidermidis, the HD motif of Csm1 is not required for DNA cleavage (Hatoum-Aslan et al. 2014). Instead, the GGDD motif in the Palm domain of Csm1 is required (HatoumAslan et al. 2014; Ramia et al. 2014b; Samai et al. 2015). Moreover, some Type III-B systems contain Cmr2 proteins lacking HD domains, such as T. thermophilus (Staals et al. 2013). In these organisms, the Type III-B system may only target RNA, or the GGDD motif may be used for DNA cleavage. More structural and biochemical studies are required to clarify the role of these two motifs (HD vs. GGDD) in the different Type III systems.

Nucleases need to be tightly regulated so they cleave only the intended substrates, as any nonspecific activity could be deleterious to the cell. The transcript-binding model (Fig. 7) provides a mechanism for both spatial and temporal regulation of DNA cleavage by the Cmr complex. Spatial regulation can be achieved by tethering the activated Cmr complex to a specific region of DNA that is actively transcribing through base pairing between the crRNA and transcript. This spatial restraint would ensure that the activated Cmr complex does not cleave at other transcription bubbles, such as those found at transcribing host genes. Thus, spatial regulation may provide a mechanism for immunity (specific cleavage of transcribing foreign DNA) rather than cell death (cleavage of transcribing host DNA). Targeting of antisense transcripts derived from host CRISPR loci, which have been observed in several species (Hale et al. 2012; Garrett et al. 2015), appears to be prevented by base paring between the $5^{\prime}$ handle of the crRNA and the complementary region of the resulting antisense transcript (Supplemental Fig. S6). Temporal regulation can be achieved by coupling DNA cleavage to the presence of an intact transcript, as, once the transcript is cleaved, the DNA nuclease activity is deactivated (Fig. 3B). Due to this coupling of DNA and RNA cleavage activities, in cells, the rate of transcript cleavage should be slower than the rate of DNA cleavage in order for efficient DNA cleavage to occur. This was not the case in our in vitro experiments, as the rate of ssDNA cleavage was somewhat slower than the rate of ssRNA cleavage (Fig. 4, cf. A, lanes $8-10$, and B, lanes $1-4)$. However, in our in vitro assay, we used ssDNA oligonucleotides as substrates. In cells, the proposed substrate is the nontemplate strand of the transcription bubble. This substrate may be a better substrate for the Cmr complex than ssDNA oligonucleotides and thus may be cleaved at a higher rate. Also, as discussed above, by targeting a transcribing region of DNA, the Cmr complex is tethered to its DNA substrate by its interactions with the nascent transcript. This tethering would increase the effective concentration of the DNA, again likely stimulating the rate of its cleavage. In addition, cleavage of an RNA transcript emerging from a transcription bubble may be slower than cleavage of an ssRNA oligonucleotide. Further experiments carefully examining these rates in the context of transcription-coupled targeting both in vitro and in vivo will be required to provide a complete mechanistic description of the system.

In summary, our studies provide biochemical evidence that the Type III-B system cleaves ssDNA in the presence of an RNA target complementary to the crRNA. These results are consistent with previous results showing that the Type III-B system protects cells from plasmid transformation in a transcription-coupled manner (Deng et al. 2013). Our data further resolve the ambiguity in activities reported for Type III systems and support a unified 
mechanism for all Type III systems in which DNA nuclease activity is coupled to transcription (Samai et al. 2015).

\section{Materials and methods}

\section{Purification of recombinant Cmr proteins}

The genes encoding Cmr1-6 were individually PCR-amplified from T. maritima genomic DNA and cloned into pET-derived vectors (Peränen et al. 1996). The vectors, pHAT4 and pHAT2, contain an $\mathrm{N}$-terminal hexahistidine tag that is cleavable or not cleavable by tobacco etch virus (TEV) protease, respectively. The vectors were transformed into T7Express cells (New England Biolabs) and grown to an $\mathrm{OD}_{600}$ of 0.3 in Luria-Bertani medium followed by induction with $0.2 \mathrm{mM}$ isopropyl- $\beta$-D-thiogalactopyranoside (IPTG) and overnight expression at room temperature. The cells were pelleted at $4500 \mathrm{rpm}$, resuspended in lysis buffer [1 M KCl, $20 \mathrm{mM}$ Tris- $\mathrm{HCl}$ at $\mathrm{pH}$ 8.0, $10 \mathrm{mM}$ imidazole, $1 \mathrm{mM}$ tris(2-carboxyethyl)phosphine (TCEP)], and disrupted using a French press. The resulting sample was centrifuged at 18,000 rpm for $45 \mathrm{~min}$ at $4^{\circ} \mathrm{C}$. The histidine-tagged $\mathrm{Cmr}$ proteins were isolated from the supernatant using a 5-mL IMAC column (Bio$\mathrm{Rad})$ charged with nickel sulfate and equilibrated with lysis buffer. After washing with at least $100 \mathrm{~mL}$ of lysis buffer, the proteins were eluted with $250 \mathrm{mM}$ imidazole-containing lysis buffer and injected onto a HiLoad 26/60 S200 size exclusion column (GE Healthcare) equilibrated with gel filtration buffer $(350 \mathrm{mM} \mathrm{KCl}$, $20 \mathrm{mM}$ Tris- $\mathrm{HCl}$ at $\mathrm{pH} 8.0,1 \mathrm{mM}$ TCEP). The above steps were carried out with Cmr proteins cloned into the pHAT2 vector. When cloned into the pHAT4 vector, histidine tag removal was accomplished by elution off of IMAC as above, followed by buffer exchange with a HiPrep 26/10 desalting column (GE Healthcare) equilibrated with gel filtration buffer and incubation with TEV protease overnight at $4^{\circ} \mathrm{C}$. The sample was reapplied to the IMAC column to remove the cleaved histidine tag and the histidine tag-containing TEV protease, and the flow-through was collected and injected onto a HiLoad 26/60 S200 size exclusion column equilibrated with gel filtration buffer. Fractions collected from the size exclusion column were analyzed by SDS-PAGE. The $\mathrm{Cmr} 2$ and $\mathrm{Cmr} 4$ mutants were created by PCR-based mutagenesis. The expression vector was amplified using phosphorylated mutagenic primers followed by self-ligation. All mutant proteins were expressed and purified using the same protocol as the respective wild-type protein.

\section{Pull-down assay}

Purified Cmr proteins that were tagged (bait) and untagged (prey) were incubated in $100 \mu \mathrm{L}$ of binding buffer $(100 \mathrm{mM} \mathrm{KCl}, 20 \mathrm{mM}$ Tris- $\mathrm{HCl}$ at $\mathrm{pH} 8.0,10 \mathrm{mM}$ imidazole, $1 \mathrm{mM}$ TCEP) for $30 \mathrm{~min}$ at $37^{\circ} \mathrm{C}$. Twenty microliters of nickel sulfate-charged IMAC resin (BioRad) equilibrated in binding buffer was added to the Cmr protein sample and incubated for $1 \mathrm{~h}$ at $4^{\circ} \mathrm{C}$ on a rotating plate. The resin was washed three times with $0.5 \mathrm{~mL}$ of binding buffer and further incubated with elution buffer (binding buffer containing $250 \mathrm{mM}$ imidazole) for $30 \mathrm{~min}$ at $4^{\circ} \mathrm{C}$. Proteins eluted from the beads were analyzed on $4 \%-20 \%$ miniprotean TGX precast gels (BioRad) stained with Coomassie.

\section{Preparation of labeled oligonucleotides}

RNA and DNA oligonucleotides were purchased from Sigma Aldrich (Supplemental Tables 1, 2). They were 5' radiolabeled with T4 polynucleotide kinase (New England BioLabs) and $\gamma^{-32}$ PATP
(Perkin Elmer) in 1× T4 polynucleotide kinase buffer for $30 \mathrm{~min}$ at $37^{\circ} \mathrm{C}$. The substrates were resolved on a denaturing polyacrylamide gel, visualized by autoradiography, excised from the gel, and placed in a $0.5-\mathrm{mL}$ solution of $0.3 \mathrm{M}$ sodium acetate overnight at $4^{\circ} \mathrm{C}$ followed by ethanol precipitation and resuspension in RNA storage solution (Ambion) for RNA or $10 \mathrm{mM}$ Tris- $\mathrm{HCl}$ (pH 8.0) and 1 mM EDTA for DNA. To generate dsDNA or dsRNA targets, labeled oligonucleotide with twice the molar excess of the complementary unlabeled oligonucleotide was incubated for $10 \mathrm{~min}$ at $95^{\circ} \mathrm{C}$ followed by slow cooling to room temperature. Complete annealing was confirmed by nondenaturing PAGE.

Radiolabeling of the $3^{\prime}$ end of ssRNA was performed as described previously (Huang and Szostak 1996). Briefly, ssRNA was annealed to a short ssDNA oligonucleotide complementary to the $3^{\prime}$ end of ssRNA, generating a TG overhang. The duplex was then incubated with a $3^{\prime}-5^{\prime}$ exonuclease-deficient Klenow fragment (New England Biolabs) and $\alpha-{ }^{32}$ PdATP (Perkin Elmer) in $1 \times$ buffer 2 (New England Biolabs) for $2 \mathrm{~h}$ at $37^{\circ} \mathrm{C}$. Following the reaction, the ssDNA was removed by denaturing PAGE.

\section{RNA cleavage assays}

Cmr2-6 was formed by pull-down (see above). Unless otherwise

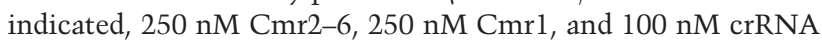
were incubated for $30 \mathrm{~min}$ at $80^{\circ} \mathrm{C}$ in reaction buffer $(100 \mathrm{mM}$ $\mathrm{KCl}, 20 \mathrm{mM}$ HEPES at $\mathrm{pH} 7,1 \mathrm{mM} \mathrm{MnCl} \mathrm{m}_{2}, 0.5 \mathrm{mM}$ ATP, $1 \mathrm{mM}$ TCEP). The reaction $\left(80^{\circ} \mathrm{C}\right)$ was initiated by addition of $1 \mathrm{nM}$ (unless otherwise indicated) radiolabeled target. The reaction was stopped at $10 \mathrm{~min}$ or the indicated time point with the addition of quencher dye $90 \%$ formamide, $2.5 \%$ glycerol, $0.01 \%$ SDS, $0.01 \%$ bromophenol blue, $0.01 \%$ xylene cyanol, $1 \mathrm{mM}$ EDTA) and heated for $10 \mathrm{~min}$ at $95^{\circ} \mathrm{C}$. Radiolabeled RNA ladders were generated with RNase T1 (New England Biolabs) or alkaline hydrolysis buffer $(50 \mathrm{mM}$ sodium carbonate at $\mathrm{pH} 9.2,1 \mathrm{mM}$ EDTA). The samples are then run on $20 \%$ polyacrylamide denaturing gels and visualized by phosphorimaging.

\section{DNA cleavage assay}

The Cmr complex was assembled with a crRNA as described above. This complex was then incubated with both a target RNA (1 nM, unless otherwise indicated) and $5^{\prime}$ labeled ssDNA (1 $\mathrm{nM}$, unless otherwise indicated) substrate in reaction buffer for $10 \mathrm{~min}$ at $80^{\circ} \mathrm{C}$. The DNA was isolated by phenol-chloroform extraction and analyzed by denaturing PAGE and autoradiography.

\section{EMSA}

$5^{\prime}$ labeled RNA target (1 nM) was incubated with $800 \mathrm{nM} \mathrm{Cmr}$ complex (wild-type and mutant complexes) loaded with $800 \mathrm{nM}$ crRNA 8.3 for $1 \mathrm{~h}$ at $4^{\circ} \mathrm{C}$ in reaction buffer. RNA cleavage was undetectable under these conditions. Following incubation, bound RNA target was resolved from the free target by electrophoresis through a $5 \%$ polyacrylamide gel. RNA was visualized by autoradiography.

\section{Acknowledgments}

We thank Jennifer M. Kavran for critical reading of the manuscript. This work was supported by National Institutes of Health grant GM097330 to S.B., and a Ruth L. Kirschstein National Institutes of Health F31 fellowship (GM105364) to M.A.E. 


\section{References}

Barrangou R, Fremaux C, Deveau H, Richards M, Boyaval P, Moineau S, Romero DA, Horvath P. 2007. CRISPR provides acquired resistance against viruses in prokaryotes. Science 315: 1709-1712.

Benda C, Ebert J, Scheltema RA, Schiller HB, Baumgärtner M, Bonneau F, Mann M, Conti E. 2014. Structural model of a CRISPR RNA-silencing complex reveals the RNA-target cleavage activity in Cmr4. Mol Cell 56: 43-54.

Bolotin A. 2005. Clustered regularly interspaced short palindrome repeats (CRISPRs) have spacers of extrachromosomal origin. Microbiology 151: 2551-2561.

Brouns SJJ, Jore MM, Lundgren M, Westra ER, Slijkhuis RJH, Snijders APL, Dickman MJ, Makarova KS, Koonin EV, van der Oost J. 2008. Small CRISPR RNAs guide antiviral defense in prokaryotes. Science 321: 960-964.

Carte J, Wang R, Li H, Terns R, Terns M. 2008. Cas6 is an endoribonuclease that generates guide RNAs for invader defense in prokaryotes. Genes Dev 22: 3489-3496.

Cocozaki AI, Ramia NF, Shao Y, Hale CR, Terns R, Terns M, Li H. 2012. Structure of the Cmr2 subunit of the CRISPR-Cas RNA silencing complex. Structure 20: 545-553.

Deltcheva E, Chylinski K, Sharma CM, Gonzales K, Chao Y, Pirzada ZA, Eckert MR, Vogel J, Charpentier E. 2011. CRISPR RNA maturation by trans-encoded small RNA and host factor RNase III. Nature 471: 602-607.

Deng L, Garrett RA, Shah SA, Peng X, She Q. 2013. A novel interference mechanism by a Type IIIB CRISPR-Cmr module in Sulfolobus. Mol Microbiol 87: 1088-1099.

Garneau JE, Dupuis M-È, Villion M, Romero DA, Barrangou R, Boyaval P, Fremaux C, Horvath P, Magadán AH, Moineau S. 2010. The CRISPR/Cas bacterial immune system cleaves bacteriophage and plasmid DNA. Nature 468: 67-71.

Garrett R, Shah S, Erdmann S, Liu G, Mousaei M, León-Sobrino C, Peng W, Gudbergsdottir S, Deng L, Vestergaard G, et al. 2015. CRISPR-Cas adaptive immune systems of the sulfolobales: unravelling their complexity and diversity. Life (Basel) 5: 783-817.

Gasiunas G, Barrangou R, Horvath P, Siksnys V. 2012. Cas9crRNA ribonucleoprotein complex mediates specific DNA cleavage for adaptive immunity in bacteria. Proc Natl Acad Sci 109: E2579-E2586.

Goldberg GW, Jiang W, Bikard D, Marraffini LA. 2014. Conditional tolerance of temperate phages via transcription-dependent CRISPR-Cas targeting. Nature 514: 633-637.

Hale CR, Zhao P, Olson S, Duff MO, Graveley BR, Wells L, Terns R, Terns M. 2009. RNA-guided RNA cleavage by a CRISPR RNA-Cas protein complex. Cell 139: 945-956.

Hale CR, Majumdar S, Elmore J, Pfister N, Compton M, Olson S, Resch AM, Glover CVC III, Graveley BR, Terns R, et al. 2012. Essential features and rational design of CRISPR RNAs that function with the Cas RAMP module complex to cleave RNAs. Mol Cell 45: 292-302.

Hale CR, Cocozaki A, Li H, Terns R, Terns M. 2014. Target RNA capture and cleavage by the Cmr Type III-B CRISPR-Cas effector complex. Genes Dev 28: 2432-2443.

Hatoum-Aslan A, Maniv I, Samai P, Marraffini LA. 2014. Genetic characterization of antiplasmid immunity through a Type IIIA CRISPR-Cas system. J Bacteriol 196: 310-317.

Huang Z, Szostak JW. 1996. A simple method for 3'-labeling of RNA. Nucleic Acids Res 24: 4360-4361.

Huber R, Langworthy TA, König H, Thomm M, Woese CR, Sleytr UB, Stetter KO. 1986. Thermotoga maritima sp. nov. repre- sents a new genus of unique extremely thermophilic eubacteria growing up to $90^{\circ} \mathrm{C}$. Arch Microbiol 144: 324-333.

Jackson RN, Wiedenheft B. 2015. A conserved structural chassis for mounting versatile CRISPR RNA-guided immune responses. Mol Cell 58: 722-728.

Jinek M, Chylinski K, Fonfara I, Hauer M, Doudna JA, Charpentier E. 2012. A programmable dual-RNA-guided DNA endonuclease in adaptive bacterial immunity. Science 337: 816-821.

Jung T-Y, An Y, Park K-H, Lee M-H, Oh B-H, Woo E. 2015. Crystal structure of the Csm 1 subunit of the Csm complex and its single-stranded DNA-specific nuclease activity. Structure 23: 782-790.

Makarova KS, Haft DH, Barrangou R, Brouns SJJ, Charpentier E, Horvath P, Moineau S, Mojica FJM, Wolf YI, Yakunin AF, et al. 2011. Evolution and classification of the CRISPR-Cas systems. Nat Rev Microbiol 9: 467-477.

Makarova KS, Anantharaman V, Aravind L, Koonin EV. 2012. Live virus-free or die: coupling of antivirus immunity and programmed suicide or dormancy in prokaryotes. Biol Direct 7: 40.

Makarova KS, Wolf YI, Alkhnbashi OS, Costa F, Shah SA, Saunders SJ, Barrangou R, Brouns SJJ, Charpentier E, Haft DH, et al. 2015. An updated evolutionary classification of CRISPR-Cas systems. Nat Rev Microbiol 13: 722-736.

Marraffini LA, Sontheimer EJ. 2010. Self versus non-self discrimination during CRISPR RNA-directed immunity. Nature 463: $568-571$.

Mojica FJM, Díez-Villaseñor CS, García-Martínez J, Soria E. 2005. Intervening sequences of regularly spaced prokaryotic repeats derive from foreign genetic elements. J Mol Evol 60: 174-182.

Mulepati S, Bailey S. 2011. Structural and biochemical analysis of nuclease domain of clustered regularly interspaced short palindromic repeat (CRISPR)-associated protein 3 (Cas3). I Biol Chem 286: 31896-31903.

Mulepati S, Bailey S. 2013. In vitro reconstitution of an Escherichia coli RNA-guided immune system reveals unidirectional, ATP-dependent degradation of DNA target. J Biol Chem 288: 22184-22192.

Osawa T, Inanaga H, Sato C, Numata T. 2015. Crystal structure of the CRISPR-Cas RNA silencing Cmr complex bound to a target analog. Mol Cell 58: 418-430.

Peränen J, Rikkonen M, Hyvönen M, Kääriäinen L. 1996. T7 vectors with a modified T7lac promoter for expression of proteins in Escherichia coli. Anal Biochem 236: 371-373.

Pourcel C. 2005. CRISPR elements in Yersinia pestis acquire new repeats by preferential uptake of bacteriophage DNA, and provide additional tools for evolutionary studies. Microbiology 151: 653-663.

Ramia NF, Spilman M, Tang L, Shao Y, Elmore J, Hale C, Cocozaki A, Bhattacharya N, Terns R, Terns M, et al. 2014a. Essential structural and functional roles of the $\mathrm{Cmr} 4$ subunit in RNA cleavage by the Cmr CRISPR-Cas complex. Cell Rep 9: 1610-1617.

Ramia NF, Tang L, Cocozaki AI, Li H. 2014b. Staphylococcus epidermidis Csm1 is a $3^{\prime}-5^{\prime}$ exonuclease. Nucleic Acids Res 42: 1129-1138.

Rouillon C, Zhou M, Zhang I, Politis A, Beilsten-Edmands V, Cannone G, Graham S, Robinson CV, Spagnolo L, White MF. 2013. Structure of the CRISPR interference complex CSM reveals key similarities with cascade. Mol Cell 52: 124-134.

Samai P, Pyenson N, Jiang W, Goldberg GW, Hatoum-Aslan A, Marraffini LA. 2015. Co-transcriptional DNA and RNA cleavage during Type III CRISPR-Cas immunity. Cell 161: 1164-1174. 
Sinkunas T, Gasiunas G, Waghmare SP, Dickman MJ, Barrangou R, Horvath P, Siksnys V. 2013. In vitro reconstitution of Cascade-mediated CRISPR immunity in Streptococcus thermophilus. EMBO J 32: 385-394.

Spilman M, Cocozaki A, Hale C, Shao Y, Ramia N, Terns R, Terns M, Li H, Stagg S. 2013. Structure of an RNA silencing complex of the CRISPR-Cas immune system. Mol Cell 52: 146-152.

Staals RHJ, Agari Y, Maki-Yonekura S, Zhu Y, Taylor DW, van Duijn E, Barendregt A, Vlot M, Koehorst JJ, Sakamoto K, et al. 2013. Structure and activity of the RNA-targeting Type III-B CRISPR-Cas complex of Thermus thermophilus. Mol Cell 52: 135-145.

Staals RHJ, Zhu Y, Taylor DW, Kornfeld JE, Sharma K, Barendregt A, Koehorst JJ, Vlot M, Neupane N, Varossieau K, et al. 2014. RNA targeting by the Type III-A CRISPR-Cas Csm complex of Thermus thermophilus. Mol Cell 56: 518-530.

Tamulaitis G, Kazlauskiene M, Manakova E, Venclovas Č, Nwokeoji AO, Dickman MJ, Horvath P, Siksnys V. 2014. Programmable RNA shredding by the Type III-A CRISPR-Cas system of Streptococcus thermophilus. Mol Cell 56: 506-517.

Taylor DW, Zhu Y, Staals RHJ, Kornfeld JE, Shinkai A, van der Oost J, Nogales E, Doudna JA. 2015. Structures of the
CRISPR-Cmr complex reveal mode of RNA target positioning. Science 348: 581-585.

van der Oost J, Westra ER, Jackson RN, Wiedenheft B. 2014. Unravelling the structural and mechanistic basis of CRISPR-Cas systems. Nat Rev Microbiol 12: 479-492.

Wang D, Landick R. 1997. Nuclease cleavage of the upstream half of the nontemplate strand DNA in an Escherichia coli transcription elongation complex causes upstream translocation and transcriptional arrest. J Biol Chem 272: 5989-5994.

Westra ER, van Erp PBG, Künne T, Wong SP, Staals RHJ, Seegers CLC, Bollen S, Jore MM, Semenova E, Severinov K, et al. 2012. CRISPR immunity relies on the consecutive binding and degradation of negatively supercoiled invader DNA by Cascade and Cas3. Mol Cell 46: 595-605.

Zebec Z, Manica A, Zhang J, White MF, Schleper C. 2014. CRISPRmediated targeted mRNA degradation in the archaeon Sulfolobus solfataricus. Nucleic Acids Res 42: 5280-5288.

Zhang J, Rouillon C, Kerou M, Reeks J, Brugger K, Graham S, Reimann J, Cannone G, Liu H, Albers S-V, et al. 2012. Structure and mechanism of the CMR complex for CRISPR-mediated antiviral immunity. Mol Cell 45: 303-313.

Zhu X, Ye K. 2015. Cmr4 is the slicer in the RNA-targeting Cmr CRISPR complex. Nucleic Acids Res 43: 1257-1267. 


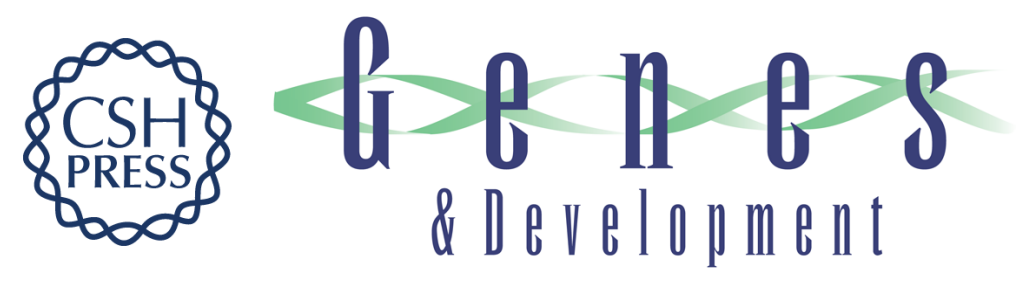

\section{RNA-activated DNA cleavage by the Type III-B CRISPR-Cas effector complex}

Michael A. Estrella, Fang-Ting Kuo and Scott Bailey

Genes Dev. 2016, 30: originally published online February 4, 2016

Access the most recent version at doi:10.1101/gad.273722.115

\section{Supplemental http://genesdev.cshlp.org/content/suppl/2016/02/04/gad.273722.115.DC1 \\ Material}

Related Content Bipartite recognition of target RNAs activates DNA cleavage by the Type III-B CRISPRCas system

Joshua R. Elmore, Nolan F. Sheppard, Nancy Ramia, et al.

Genes Dev. February , 2016 30: 447-459

References This article cites 47 articles, 12 of which can be accessed free at: http://genesdev.cshlp.org/content/30/4/460.full.html\#ref-list-1

Articles cited in: http://genesdev.cshlp.org/content/30/4/460.full.htmI\#related-urls

Creative This article is distributed exclusively by Cold Spring Harbor Laboratory Press for the first Commons six months after the full-issue publication date (see

License http://genesdev.cshlp.org/site/misc/terms.xhtml). After six months, it is available under a Creative Commons License (Attribution-NonCommercial 4.0 International), as described at http://creativecommons.org/licenses/by-nc/4.0/.

Email Alerting Receive free email alerts when new articles cite this article - sign up in the box at the top Service right corner of the article or click here.

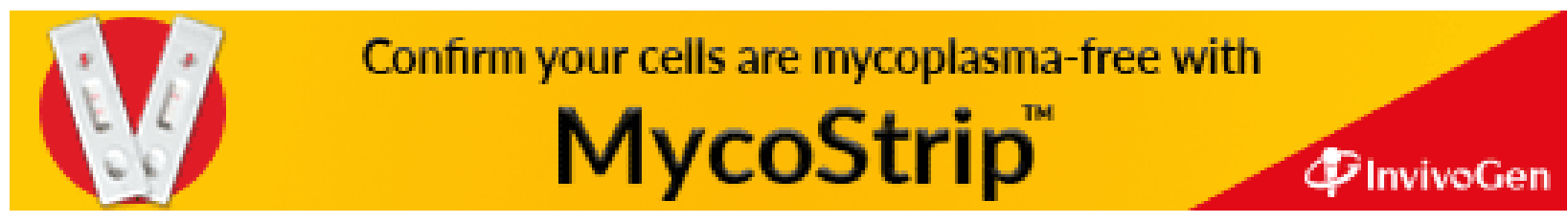

